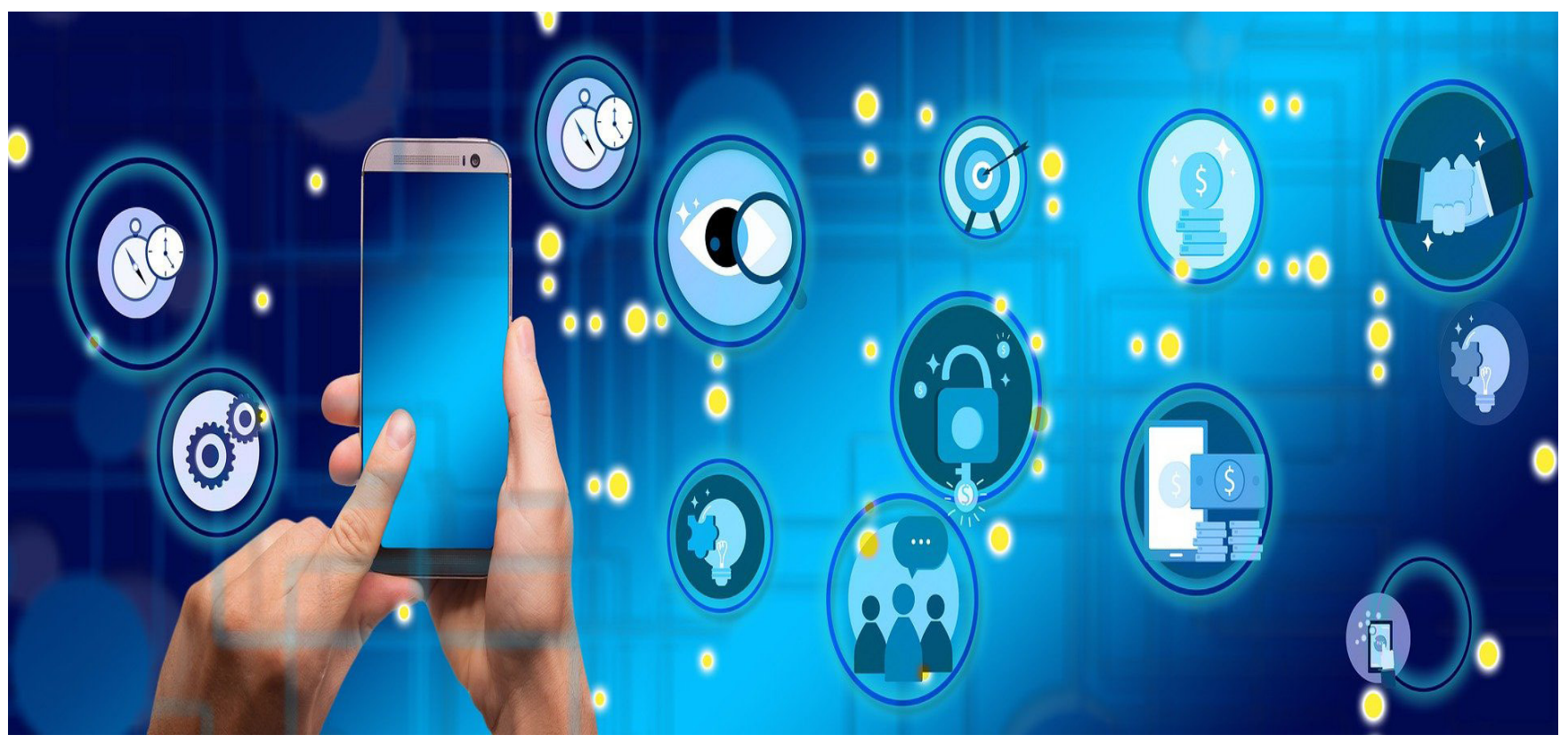

\title{
Planes de marketing en las pymes sector calzado de la ciudad de Cúcuta
}

Marketing plans in the footwear sector SMEs of the city of Cúcuta

\section{Luis Alfredo Bustamante-Díaz}

Estudiante de negocios. la_bustamante@fesc.edu.co

Fundación de Estudios Superiores Comfanorte, Cúcuta, Colombia

Darly Karolayn Díaz-Machuca

Estudiante de negocios. Fundación de Estudios Superiores Comfanorte, Cúcuta, Colombia

Wilker David Pinzón-Ayala

Especialista Gestión Pública, gp_a_aro@fesc.edu.co Fundación de Estudios Superiores Comfanorte - Fesc, Cúcuta, Colombia
Nathaly Melissa Zambrano-Martínez

Estudiante de negocios. Fundación de Estudios Superiores

Comfanorte, Cúcuta, Colombia

Maria de los Angeles Barrientos-Rosales Estudiante de Financiara, est_ma_barrientos@fesc.edu.co Fundación de Estudios Superiores Comfanorte, Cúcuta, Colombia 
Cómo citar: L.A. Bustamante-Díaz, D.K. Díaz-Machuca, W.D. Pinzón-Ayala, N.M. ZambranoMartínez, M. de los Angeles Barrientos-Rosales, "Planes de marketing en las pymes sector calzado de la ciudad de Cúcuta". Reflexiones contables (Cúcuta), vol. 3, no. 2, 8-19.

\section{Resumen}

El propósito de este trabajo fue conocer como manejan los planes de marketing las empresas del calzado en la ciudad de Cúcuta, esto por cuanto las bondades que brinda el marketing es fundamental para la competitividad de la organización y así la de su desarrollo, con esto se tiene que la utilización de estas herramientas de publicidad fomentan a una empresa a elevar sus actividades y a darse a conocer en los diferentes contextos para posicionarse, y más el sector del calzado siendo un sector importante en Norte de Santander que necesita mejorar sus desempeños publicitarios. De la misma manera esta investigación se realizó bajo un enfoque cuantitativo ya que se aplicó una encuesta en el sector para poder develar de que manera era la utilización del marketing en las pymes del sector.

Palabras Clave: Marketing, Pymes, fabricas, Planificación.

\section{Abstract}

The purpose of this work was to know how footwear companies in the city of Cúcuta handle the marketing plans, this because the benefits that marketing offers is fundamental for the competitiveness of the organization and thus that of its development, with this The use of these advertising tools has to encourage a company to increase its activities and make itself known in different contexts to position itself, and more so the footwear sector is an important sector in Norte de Santander that needs to improve its advertising performance. In the same way, this research was carried out under a quantitative approach since a survey was applied in the sector to be able to reveal how the use of marketing was in SMEs in the sector.

Keywords: Marketing, SMEs, factories, Planning. 
Planes de marketing en las pymes sector calzado de la ciudad de Cúcuta

10

\section{Introducción}

Los microempresarios de la ciudad de Cúcuta no cuentan con el conocimiento necesario para desarrollar un plan de marketing, incluso algunos creen que es innecesarios realizar una inversión sobre este tema. El principal motivo por el cual se debe realizar un plan de marketing, es porque ayuda a la consecución de los objetivos de la empresa, que están relacionados con el mercado, como, por ejemplo: captación de nuevos clientes, creación de marca, fidelización de clientes, aumento de la facturación o el incremento de la participación en el mercado. En definitiva, el plan de marketing, debería ser un documento de obligada realización para todas las empresas, donde el tamaño o el sector al que pertenecen deberían dejar de ser excusas para no realizarlo. (García 2018).

Los microempresarios de la ciudad de Cúcuta se están viendo afectados en la parte económica de sus empresas debido a la falta de conocimiento o implementación de estrategias que puedan aportar para el cumplimiento de sus objetivos y metas trazadas. Por medio del siguiente trabajo queremos dar a conocer la importancia de implementar un Plan de Marketing en microempresas debido a que es una herramienta fundamental para alcanzar los objetivos, permitiendo una preparación informada que se puede aplicar tanto a nuevos productos como al aumento de ventas de productos existentes. (Marulanda 2018).

Por lo cual es necesario conocer y aplicar las nuevas estrategias para que incrementen sus ventas, brindando diferenciación en sus productos y servicios, entre otros factores que ayudaran a posicionar la marca en el mercado y sean reconocidos.

Con estos interrogantes nos permitimos analizar el uso e implementación del marketing en microempresas de la ciudad de Cúcuta como mecanismo de impulso y fortalecimiento de inversión en el sector. (Torres 2009).

Marketing es una palabra proveniente de la lengua inglesa a pesar de esta intrínseca en la cultura mundial. En inglés, market significa merca y marketing puede ser traducido como mercadotecnia o mercadeo, lo que es, en últimas, un estudio de las causas, objetivos y resultados que son generados a través de las diferentes formas en cómo lidiamos con el mercado.

"Marketing es la ciencia y el arte de explorar, crear y entregar valor para satisfacer las necesidades de un mercado objetivo con lucro. El Marketing identifica necesidades y deseos no realizados. Define, mide y cuantifica el tamaño del mercado identificado y el lucro potencial." - Philip Kotler

Según la American Marketing Association, la definición del término es la siguiente: "El Marketing es una actividad, conjunto de instituciones y procesos para crear, comunicar, entregar y cambiar las ofertas que tengan valor para los consumidores, clientes, asociados y sociedades en general". (Solano 2017).

Es decir, el Marketing es una serie de estrategias, técnicas y prácticas que tienen como principal objetivo, agregar valor a las determinadas marcas o productos con el fin de atribuir una importancia mayor para un determinado público objetivo, los consumidores.

Se engaña quien cree que el Marketing tiene solo como objetivo vender algo. Este concepto se profundiza en todo lo que envuelve este proceso, sea como la producción, logística, comercialización y post venta del producto/ servicio. 


\section{Plan de Marrketing}

El plan de marketing es un documento que forma parte de la documentación de la planificación estratégica de una empresa. El plan de marketing sirve para recoger los objetivos y estrategias, además de las acciones que van a ser necesario realizar para conseguir estos objetivos (kotler, 2000). Es la bitácora mediante la cual la empresa establece que Objetivos en términos comerciales quiere alcanzar y que debe hacer para alcanzarlos.

- ¿Para qué nos sirve?

Conocer con certeza debilidades, fortalezas, oportunidades y amenazas

Generar una disciplina en torno a la cultura de planeary supervisar las actividades de marketing de manera formal, sistemática y permanente

Aclarar el pensamiento estratégico y definir prioridades en la asignación de recursos.

\section{Análisis del mercado}

En este tema se empezará a describir teorías correspondientes al mercado, los tipos de mercados, segmentación de mercado " sus características, también se hará énfasis en detallar teorías relacionada con la publicidad de las empresas, los tipos de publicidad, los medios publicitarios que debe utilizar la Empresa. (Taquia 2015).

\section{Concepto de mercado}

Según Philip Kotler (1995: pag.462) Mercado "es el conjunto de todos los compradores reales potenciales de un producto o un servicio". Cada una las personas que buscan satisfacer sus necesidades comprando $\mathrm{X}$ o $\mathrm{Y}$ producto/ servicio hacen parte de este conjunto, cada una de estas personas están dispuesta hacer un intercambio a cambio de lo que ellos quieren, el mercado oferente siempre dependerá de la cantidad de personas que demande dicho producto o servicio.

También mercado se puede definir de la siguiente manera "Es el lugar donde se lleva a cabo el intercambio entre vendedor " comprador" (Llanos 2018), en este intercambio los vendedores buscan que el usuario demande cada vez más el servicio para que la empresa se pueda posicionar cada vez más en el mercado buscando siempre satisfacer las necesidades del cliente, por lo cual ante elcreciente aumento de los individuos que conforman el mercado global, los gerentes deben tener identificado su mercado meta hacia el cual desean proyectarse por ende mercado meta, de acuerdo con Ricardo Schlesinger, María W., \& Useche, María C. (2005). " Es el conjunto de personas hacia quienes va dirigidos todos los esfuerzos de mercadotecnia, es decir el que cumple con todas las características del segmento del mercado", que se desea cubrir al cual como empresarios podemos satisfacer sus demandas y necesidades.

\section{Estrategias de marketing}

Las estrategias de marketing se realizarán de acuerdo con los objetivos planteados, lograr que nuestros microempresarios del sector de fabricación y distribución de calzado de una parte de la ciudad, conozcan e identifiquen la importancia de aplicar los planes de marketing, debido a que es una gran oportunidad de incrementar sus ventas, dar a conocer la marca en la ciudad y obtener una buena rentabilidad. (Barrientos 2019)

\section{- Concepto de estrategia}

Las estrategias son patrones o planes que integra las principales metas "políticas de una organización, " a la vez, establece la secuencia coherente de las acciones a realizar. Una estrategia debidamente formulada ayuda a 
Planes de marketing en las pymes sector calzado de la ciudad de Cúcuta

12

poner en orden " asignar, con base tanto en sus atributos como en sus deficiencias internas, los recursos de una organización, con el fin de lograr una situación viable" original, así como anticipar los posibles cambios en el entorno " las acciones imprevistas de los oponentes. (Alvares 2015).

\section{Hipótesis de la investigación}

Para esta investigación se postularon dos hipótesis, las cuales siendo una que los microempresarios de la ciudad no aplican planes de marketing porfalta de conocimiento y de interés y también que que no aplican las estrategias y herramientas tales como las redes, los influencer, publicidad por medio de carteles, impresiones, tarjetas, producto asertivo con precios adecuados para así captar la atención de nuevos y antiguos clientes.

\section{Materales y métodos}

El enfoque que concierne a nuestra investigación será cuantitativo. Pretende utilizar el análisis y relación de datos con el objetivo de responder tanto a los interrogantes de investigación como a las hipótesis anteriormente planteadas. Este enfoque confía en mediciones numéricas, el conteo y el del análisis estadístico para proponer patrones de comportamiento poblacional para acotar la información. Efectúa las mediciones de las variables que contemplan la hipótesis y aplica diferentes técnicas estadísticas para robustecer los resultados.

El alcance de la investigación será descriptivo. El cual depende plenamente del problema de investigación. Se recurre al estudio descriptivo para analizar cómo es y cómo se manifiesta un evento, comunidad, fenómeno, hecho, contexto, situación y cada uno de sus elementos. Busca puntualizar propiedades, características y perfiles del fenómeno a analizar. Pretende medir o recolectar información de los conceptos o variables del estudio para su explicación, pero no indica cómo se relacionan entre ellas. Desde el punto de vista científico describir consiste en: recopilar datos.

En la investigación se trabajo con una población los datos recabados corresponden a: 20 de los 120 microempresarios de fabricación y venta de calzado de la ciudad de Cúcuta los anteriores. Tales elementosdatos no dependen de la probabilidad en su selección, sino de causas relacionadas con las características de los elementos que integran los datos del estudio. Por tales razones, la población de la investigación es no probabilística o dirigida. La característica principal de estos elementos, radica en que a partir de ellos se pueden hacen inferencias de la población. (Orengo 2012).

\section{Resultados y análisis}

\section{Análisis de resultados de la encuesta}

Resultados obtenidos de la encuesta aplicada a los microempresarios del Norte Santander donde la muestra de estudio es conformada por 20 microempresarios de producción y comercialización de calzado de la ciudad de Cúcuta, arroja los siguientes resultados: 
1. ¿Con que frecuencia has escuchado a otros microempresarios hablar de los planes de marketing para el negocio?

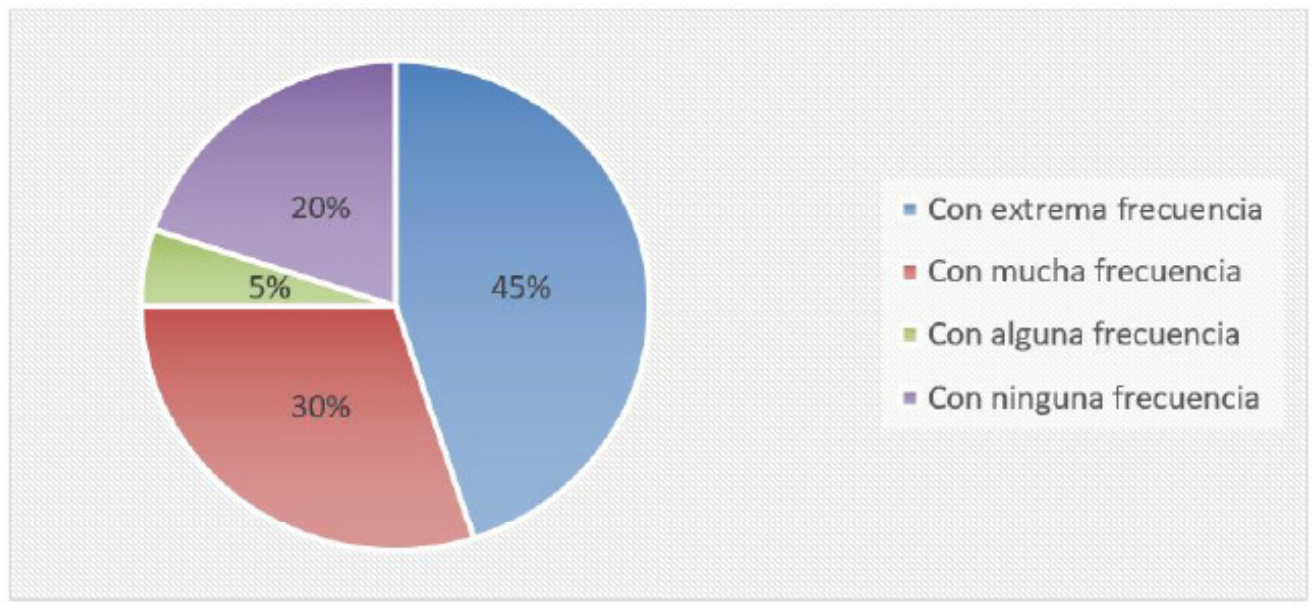

Fuente: Elaboración propia

Los microempresarios escuchan hablar con mucha frecuencia sobre los planes de marketing aun así al parecer no le prestan mucha atención.

2. ¿Sabes cuál es la función principal de los planes de marketing?

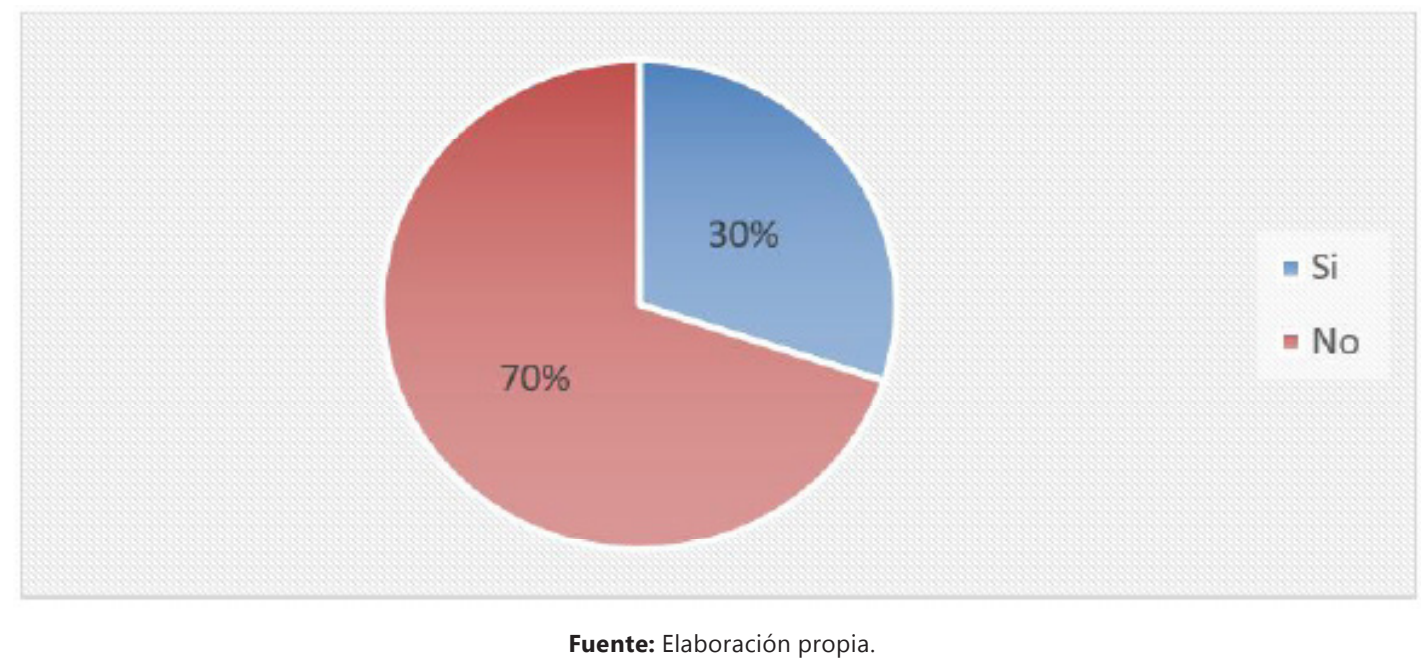

En un $70 \%$ los microempresarios no saben cuál es la función de los planes de marketing, lo que nos daría a entender que por ello es que quizá no le prestan mucha atención cuando le hablan del tema. 
Planes de marketing en las pymes sector calzado de la ciudad de Cúcuta

\section{4}

3. ¿Cuáles son las señales de que es necesario hacer algo para mejorar el marketing?

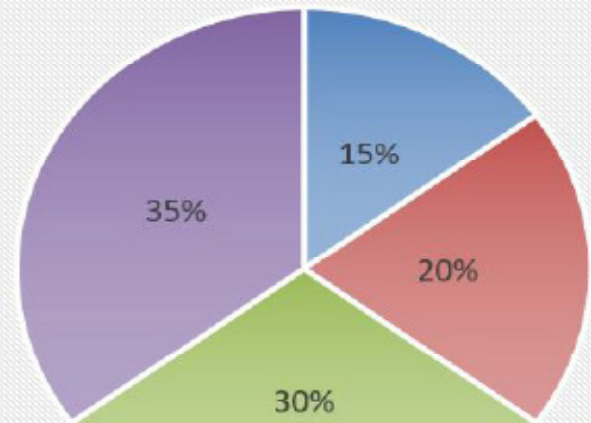

- Las ventas estan estancadas y la empresa necesita una estrategia de crecimiento

- Las ventas tienen temporadas altas y bajas que es necesario equilibrar

- Las ventas estan bien, pero los márgenes son demasiado bajos

- Las ganancias provenientes de ciertos productos son demasiado bajas.

Fuente: Elaboración propia

Si las ventas están bien, pero los márgenes son demasiados bajos, o si las ganancias provenientes de ciertos productos son demasiado bajas; son señales de que tu como microempresario debes hacer algo para mejorar el marketing.

4. ¿Qué tan probable es que utilice planes o estrategias de marketing para su negocio?

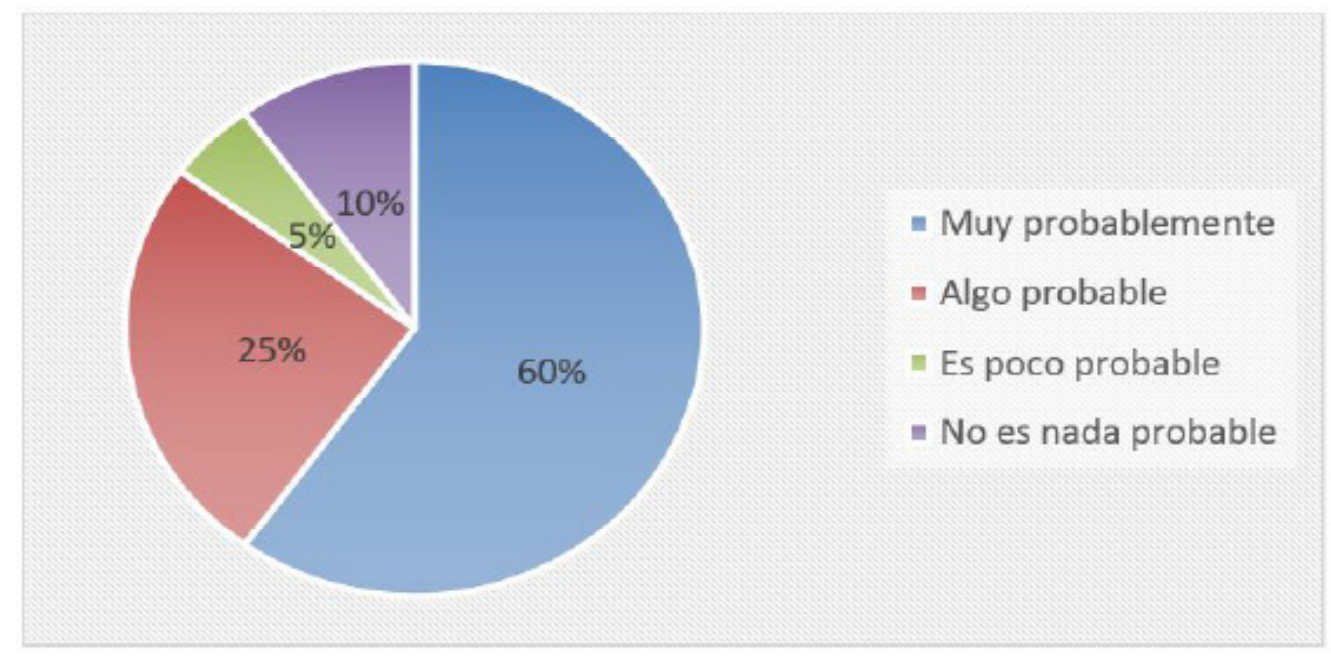

Fuente: Elaboración propia

En un $60 \%$ es muy probable que los microempresarios utilicen o implementen estrategias de marketing, después de conocer que es, les interesa, solo les falta conocer un poco mas del tema. 
5. ¿Si tuvieres la posibilidad de aplicar planes o estrategias de marketing a tu negocio, lo aplicarías?

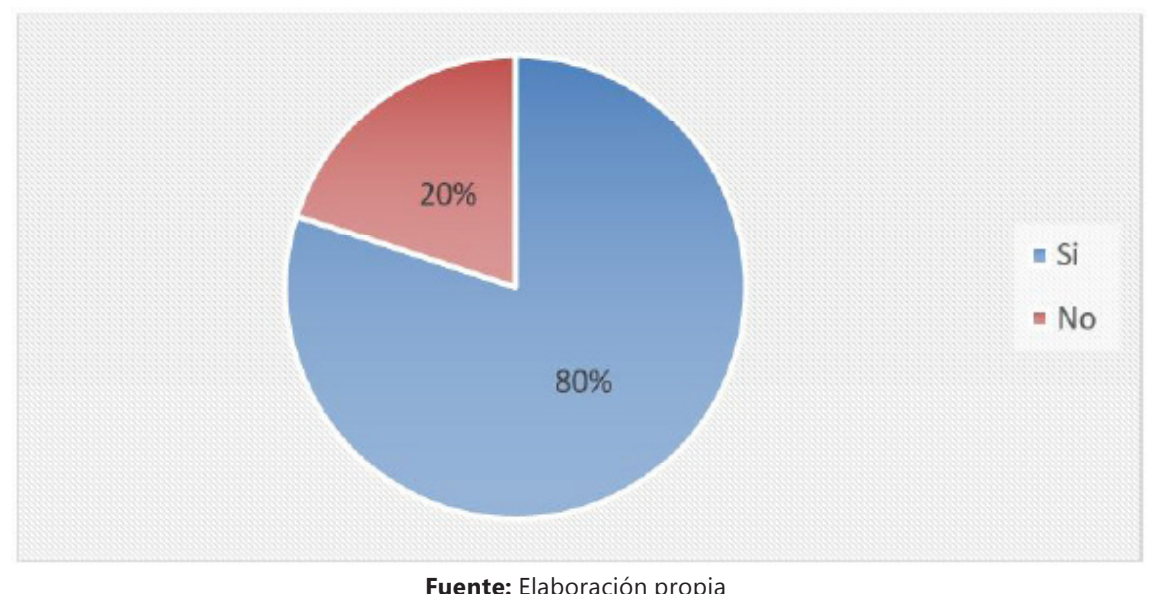

Fuente: Elaboración propia

En un $80 \%$ si los microempresarios tuvieran la oportunidad de aplicar planes de marketing lo harían.

6. Cual crees tú, ¿qué sería el factor más relevante para no llevar un plan de marketing de tu negocio?

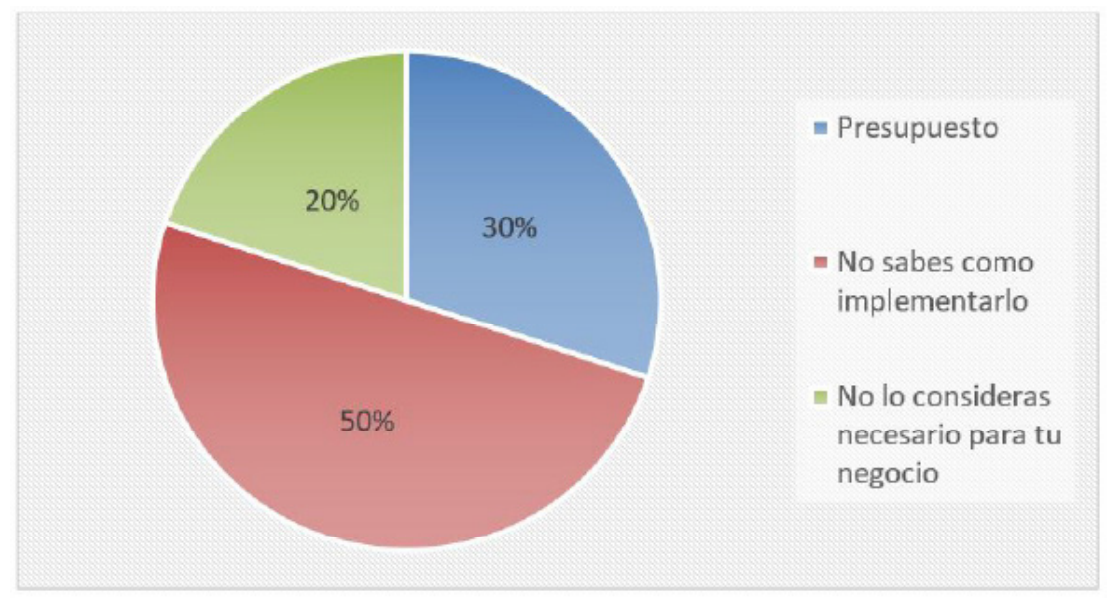

Fuente: Elaboración propia

La pregunta 5 nos arroja que si ellos tuvieran la oportunidad de implementar una nueva estrategia de marketing para su negocio lo harían, pero en un $50 \%$ no lo implementan porque no saben cómo implementarlo. 
Planes de marketing en las pymes sector calzado de la ciudad de Cúcuta

16

7. En general, ¿está satisfecho/a o insatisfecho/a con los resultados obtenidos por su negocio?

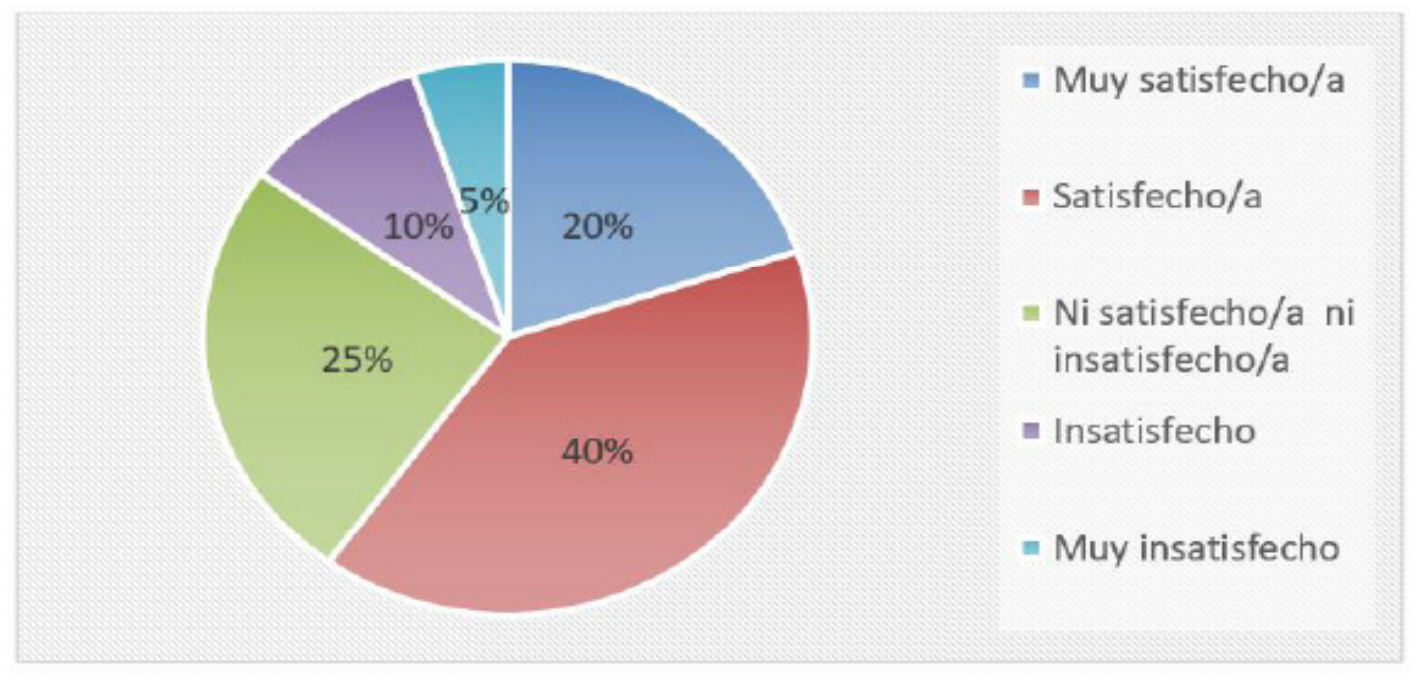

Fuente: Elaboración propia

En general los microempresarios se sienten satisfechos con los resultados de su negocio.

8. Díganos cuál es la razón por la que no le atrae o no ha adquirido el servicio:

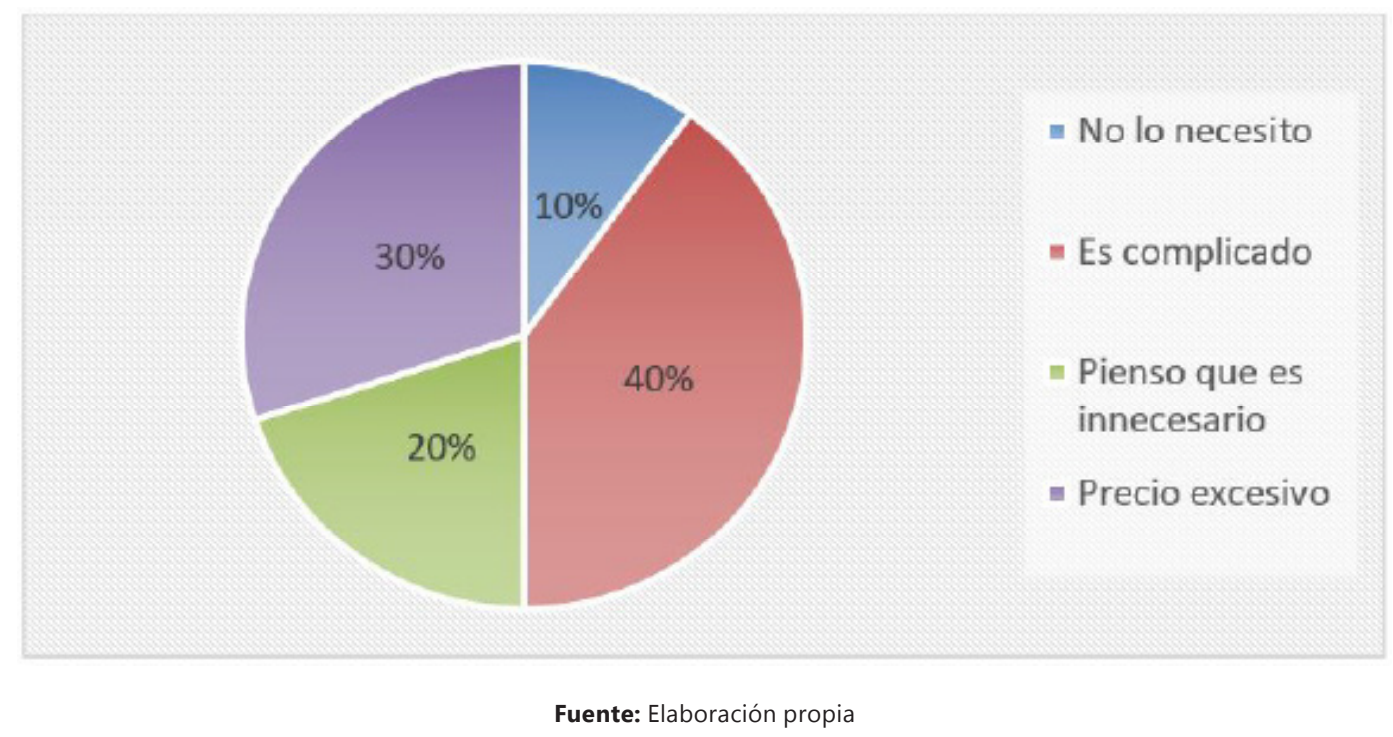

Los microempresarios en un $40 \%$ no han adquirido el servicio por que se les hace complicado, esto quiere decir aún no tienen la información necesaria para aplicarlo. 
9. ¿Cuánto estaría dispuesto a pagar por un diseño de un plan de marketing para su empresa?

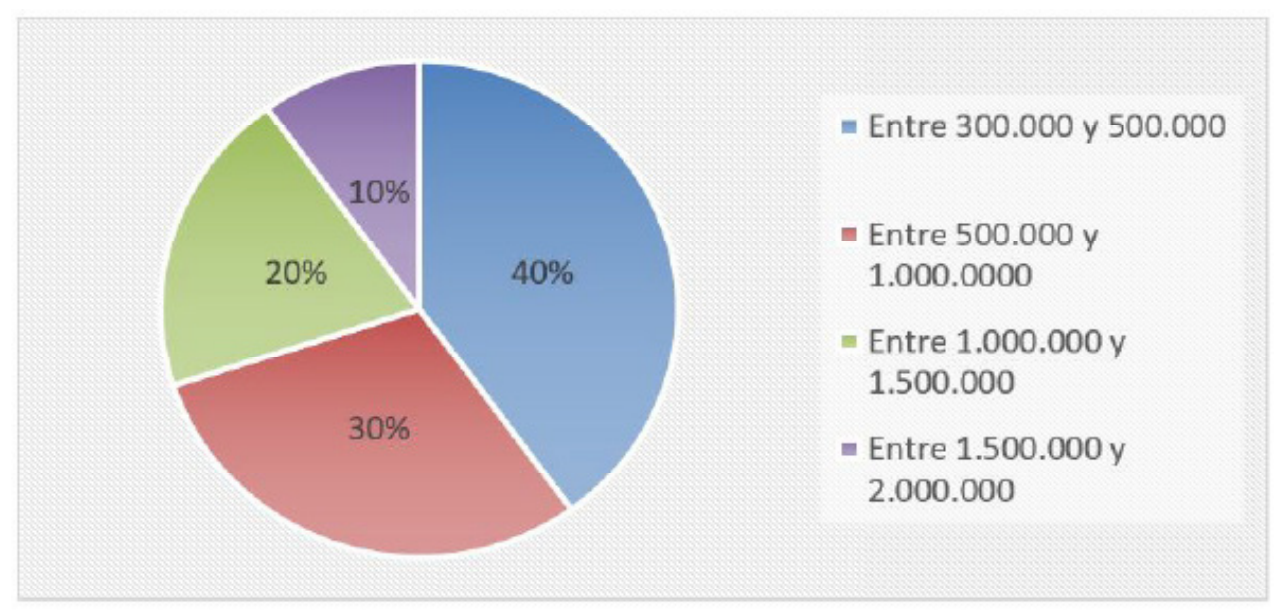

Fuente: Elaboración propia.

De los 20 microempresarios encuestados el 30\% pagaría un valor medio entre 500.000 y 1.000.000 para implementar su estrategia de marketing e incrementar las ventas en su negocio. Si les interesa invertir en el servicio.

10. Partiendo de la base que el servicio le satisfaga, ¿lo compraría?

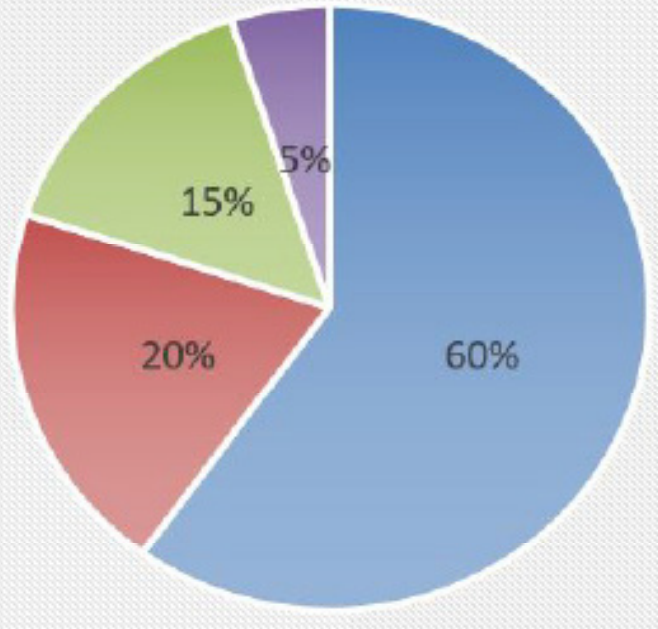

- Si, pero dejaría pasar un tiempo.

- Puede que lo comprase o puede que no.

- No, no creo que lo comprase.

- No, no lo compraría

Fuente: Elaboración propia

De los 20 microempresarios de calzado, el 60\% si comprarían el servicio, aunque dejarían pasar un tiempo. Puede ser en estos momentos por la situación o porque aún no lo presupuestan en sus gastos mensuales. 
18

\section{Conclusiones}

Con el presente trabajo se evidencia la falta de conocimiento que tienen las empresas del sector del calzado sobre el marketing, y de su potencial para mejorar la competitividad de la empresa, es asi que con base a las hipótesis planteadas los microempresarios de la ciudad no aplican planes de marketing por falta de conocimiento, mostrando con ello que no se aplican estrategias adecuadas para los fines que promete los escenarios del marketing, y el uso de herramientas tales como las redes, los influencer, publicidad por medio de carteles, impresiones, tarjetas, producto asertivo con precios adecuados para así captar la atención de nuevos y antiguos clientes.

\section{Referencias}

Álvarez Marín, Nelson, \& Trujillo Trujillo, John. (2015). Cooperación e integración en la gestión de la cadena de suministros en pymes del calzado en la ciudad de Bogotá. Dimensión Empresarial, 13(1), 147-164. https://doi.org/10.15665/rde.v13i1.343

Barrientos-Monsalve, E. J., Rosales-Nuñez, N. A., Rojas Suarez, J. P., \& Barrientos Rosales, M. de los Ángeles. (2019). La teoría fundamentada como herramienta para investigar los escenarios del marketing. Mundo FESC, 9(17), 44-48. Recuperado a partir de https://www.fesc.edu.co/Revistas/ OJS/index.php/mundofesc/article/view/361

Barrientos Rosales, M. de los A., \& Duarte Rey, D. M. (2020). Marketing para el desarrollo económico y social de las Zonas Especiales de Norte de Santander - Decreto 2112 de 2019. Sostenibilidad, Tecnología Y Humanismo, 11(2), 31-37. https://doi.org/10.25213/22161872.53

García, O. A. L., \& Hernández, E. N. P. (2018).
Aplicación de las Tecnologías de Información y comunicación en los procesos de innovación empresarial. Revisión de la literatura. I+ D REVISTA DE INVESTIGACIONES, 11(1), 144-152.

García Guiliany, J., Paz Marcano, A., \& Cantillo Campo, N. (2019). Estrategia y habilidades para la competitividad: caso de pymes del sector construcción en Barranquilla. Aglala, 10(1), 312-339. Disponible en http://revistas. curnvirtual.edu.co/index.php/aglala/article/ view/1349

Llano Restrepo, P. (2018). Entrenamiento en el área del servicio al cliente en los centros de experiencia Movistar Cartagena. Conocimiento Global, 3(1), 70-82. Recuperado a partir de http://conocimientoglobal.org/revista/index. php/cglobal/article/view/29

Mendoza-Ferreira, O. (2013). Usos y beneficios de la investigación de mercados: nuevas tendencias e influencias de la interactividad. Aibi Revista De investigación, administración E ingeniería, 1(1). https://doi.org/10.15649/2346030X.538

Marulanda Ascanio, C., Bastos Osorio, L. M., Gómez Mina, L., \& Barrientos Monsalve, E. J. (2019). Analysis of the main labor market indicators by gender in Cúcuta in the period 2010-2017. Mundo FESC, 8(15), 90-97. Recuperado a partir de https://www.fesc.edu. co/Revistas/OJS/index.php/mundofesc/article/ view/363

Marín López, J. C., \& López Trujillo, M. (2020). Análisis de datos para el marketing digital emprendedor. Caso de estudio del Parque de Innovación Empresarial de Manizales. Universidad \& Empresa, 22(38), 65-78.

Torres, Milagros (2009). Comunicación de mercadeo integral para las PyME del sector calzado, cuero y textil. Visión Gerencial, ( ),230- 
242.[fecha de Consulta 16 de Marzo de 2021]. ISSN: 1317-8822. Disponible en: https://www. redalyc.org/articulo.oa?id=4655/465545882001

Taquía Gutiérrez, José Antonio (2015). Retail marketing para desarrollar mercados emergentes. Ingeniería Industrial, (33),133155.[fecha de Consulta 16 de Marzo de 2021]. ISSN: 1025-9929. Disponible en: https://www. redalyc.org/articulo.oa?id=3374/337443854006

KOTLER, P.; CÁMARA, D. y GRANDE, I. (1995)

Dirección de Marketing, $8^{\text {a }}$ Edición, Prentice.

Herdforshire.

KOTLER, P.; CÁMARA, D.; GRANDE, I. y CRUZ, I. (2000). Dirección de Marketing, Edición del milenio, Prentice Hall, Madrid.

Solano-Becerra, E. (2017). Análisis de los potenciales efectos en el reconocimiento de los activos de las pequeñas empresas de CúcutaColombia por la implementación de la NIIF para Pymes. Respuestas, 22(2), 116-130. https:// doi.org/10.22463/0122820X.1212

Schlesinger, María W., \& Useche, María C. (2005). Mercadeo en las pymes y cooperativas en el estado Zulia. Revista Escuela de Administración de Negocios, (53),126-135. [fecha de Consulta 16 de Marzo de 2021]. ISSN: 0120-8160. Disponible en: https://www. redalyc.org/articulo.oa?id=206/20605309

Orengo, Karen L. (2012). Internacionalización y orientación empresarial. Una perspectiva de redes: cuatro casos de pymes puertorriqueñas. AD-minister, (21), 33-54. Retrieved March 16, 2021, from http://www.scielo.org.co/ scielo.php?script $=$ sci_arttext\&pid=S169202792012000200004\&lng=en\&tlng=es. 